\title{
Members of a mammalian SNARE complex interact in the endoplasmic reticulum in vivo and are found in COPI vesicles
}

\author{
Sophie Estelle Verrier ${ }^{\mathrm{a}}$, Matthias Willmann ${ }^{\mathrm{a}}$, Dirk Wenzel ${ }^{\mathrm{a}}$, Ulrike Winter ${ }^{\mathrm{a}}$, \\ Gabriele Fischer von Mollard ${ }^{\mathrm{b}, *}$, Hans-Dieter Söling ${ }^{\mathrm{a}, 1}$ \\ ${ }^{a}$ Department of Neurobiology, Max-Planck-Institute of Biophysical Chemistry, Göttingen \\ ${ }^{\mathrm{b}}$ Universität Bielefeld, Fakultät für Chemie, Biochemie III, Universitätsstraße 25, D-33615 Bielefeld, Germany
}

Received 27 March 2008; received in revised form 19 June 2008; accepted 3 July 2008

\begin{abstract}
Retrograde traffic between the Golgi apparatus and the endoplasmic reticulum (ER) is largely mediated by COPIcoated transport vesicles. In mammalian cells, retrograde traffic can pass through an intermediate compartment. Here, we report that the mammalian soluble $N$-ethylmaleimide-sensitive factor (NSF) attachment receptor (SNARE) proteins mSec22b, mUse1/D12, mSec20/BNIP1, and syntaxin 18 form a quaternary SNARE complex. Fluorescence resonance energy transfer (FRET) experiments prove that these interactions occur in the ER of living cells. In addition, mUse1/D12 and mSec20/BNIP1 form homo-oligomers in vivo. Furthermore, we show that mSec22b, mUse1/D12, mSec20/BNIP1, and syntaxin 18 are recruited into COPI-coated vesicles formed in vitro. Immunogold electron microscopy confirmed that these SNARE proteins colocalize with the KDEL receptor ERD2 in COPI-coated vesicles. Moreover, both FRET and immunoprecipitation experiments reveal interactions of these SNAREs with both ERD2 and COPI subunits. We conclude that the SNAREs described here are sorted via interaction with components of the COPI-dependent budding complex into Golgi-to-ER retrograde COPI vesicles and function in retrograde transport from the Golgi to the ER Golgi intermediate compartment (ERGIC) or the ER.
\end{abstract}

(C) 2008 Elsevier GmbH. All rights reserved.

Keywords: SNARE proteins; Live-cell FRET; COPI vesicles; KDEL receptor; Golgi-to-ER transport; Syntaxin 18; Use1; Sec20

\section{Introduction}

Intracellular membrane trafficking allows for dynamic flux of proteins and lipids between different compartments. This is largely mediated by the budding of vesicles from a donor compartment, followed by their transport to an acceptor compartment membrane and

\footnotetext{
*Corresponding author. Tel.: + 495211062081 ; fax: + 495211066014

E-mail address: gabriele.mollard@uni-bielefeld.de (G.F. von Mollard).

${ }^{1}$ Deceased.
}

subsequent fusion. The mechanisms involved in vesicular transport are conserved in evolution (Bennett and Scheller, 1993). SNARE proteins are thought to determine, together with Rab proteins and tether complexes, the specificity of intracellular fusion steps (Chen and Scheller, 2001; McNew et al., 2000). Fusion of transport intermediates requires the participation of SNAREs on transport vesicles and target membranes. Complex formation between SNAREs on opposing membranes via the conserved SNARE motifs is a key event in membrane fusion (Jahn et al., 2003; Weimbs et al., 1997). Assembly results in a trans-SNARE 
complex, in which the transmembrane domains are anchored in different membranes, and both membranes approach each other. SNAREs are aligned in parallel in a cis-complex after fusion. Structural studies allowed for a subdivision of SNAREs into four different groups (R-, Qa-, Qb-, and Qc-SNAREs) according to similarities in their amino acid sequence (Fasshauer et al., 1998). All well characterized SNARE complexes are composed of four different SNARE helices, one from each group (Antonin et al., 2002; Sutton et al., 1998). Combinatorial use of various members of the R-, Qa-, Qb-, and Qc-SNAREs gives rise to a wide array of SNARE complexes. In mammalian cells the composition of many complexes as well as the trafficking steps in which they operate are not known (Chen and Scheller, 2001; Hay, 2001). Subcellular localization is not sufficient to predict function because newly-made and recycling SNAREs are transported as cargo to the compartment in which their function is required.

Cyclic transport processes involving SNAREmediated organelle fusion serve to maintain size and shape of intracellular compartments. This is particularly evident for the transport between the ER and the cisGolgi where anterograde and retrograde traffic balance each other. In mammalian cells, COPII-coated vesicles bud from ER exit sites and form the ERGIC, vesicular tubular clusters or the cis-Golgi network by homotypic fusion (Altan-Bonnet et al., 2004; Appenzeller-Herzog and Hauri, 2006). The ERGIC is thought to mature to the Golgi by heterotypic fusion with retrograde intraGolgi COPI vesicles. However, new evidence suggests that the ERGIC could be a stable compartment. COPIcoated transport intermediates (vesicles or tubules) mediate intra-Golgi and Golgi-to-ER retrograde transport (Kreis et al., 1995; Orci et al., 1997). Retrograde traffic can passage through the ERGIC (Ben-Tekaya et al., 2005; Marra et al., 2001; Volpicelli-Daley et al., $2005)$. The COPI coatomer $(700 \mathrm{kDa})$ is a cytoplasmic complex of seven protein subunits: $\alpha$-COP, $\beta$-COP, $\beta^{\prime}$-COP, $\gamma$-COP, $\delta$-COP, $€$-COP, and $\zeta$-COP (Hara-Kuge et al., 1994). In particular, occupied KDEL receptors and membrane proteins with a cytoplasmic C-terminal KKXX motif interact with COPI and are sorted into budding retrograde vesicles in the cis-Golgi (Aoe et al., 1998; Bremser et al., 1999; Cosson et al., 1998). The KDEL receptor, also called ERD2, binds ER-resident proteins with a C-terminal KDEL (HDEL) motif which have escaped from the ER and allows for their transport back to the ER (Hardwick and Pelham, 1992).

In the yeast Saccharomyces cerevisiae, the Golgi is not organized into stacks and there is no evidence for an ERGIC. In yeast cells, the anterograde ER-to-Golgi transport via COPII-coated vesicles requires the R-SNARE Sec22p, and the Q-SNAREs Betlp (Qc), Bos1p (Qb), and Sed5p (Hardwick and Pelham, 1992; Newman et al., 1990). Sec22p recycles from the Golgi back to the ER, and this recycling involves sorting into COPI vesicles and the ER SNARE proteins Ufelp and Sec20p (Ballensiefen et al., 1998; Lewis et al., 1997). Recently, Dilcher et al. (2003) identified Uselp (also called Slt1p (Burri et al., 2003)) which forms a SNARE complex with Sec20p, Ufe1p and Sec22p, and participates in Golgi-to-ER retrograde traffic.

In mammalian cells, a complex containing SNAREs showing sequence homologies to yeast SNAREs involved in ER-to-Golgi transport has been described $(\mathrm{Xu}$ et al., 2000). It contains syntaxin 5 (Qa), membrin (Qb), Bet1 (Qc), and Sec22b (R) and mediates homotypic fusion of ER-derived COPII vesicles to form the ERGIC (Zhang et al., 1997, 1999). A mammalian homolog of Ufelp was already known as syntaxin 18 and interacts with mSec22b (Hatsuzawa et al., 2000). While this work was in progress, Hirose et al., 2004, Nakajima et al., 2004 and Okumura et al., 2006 reported the sequences of the respective human orthologs of Sec20p and Use1p as well as mouse Use1p which they called BNIP1 and p31 or D12, respectively. Nakajima et al. (2004) showed that mSec22b, syntaxin 18, $\mathrm{p} 31 / \mathrm{mUse} 1$ and BNIP1/mSec20 form a SNARE complex, which is dissociated upon ATP- $\mathrm{Mg}^{2+}$ treatment in the presence of NSF and $\alpha$-SNAP. BNIP1 is required for an intact ER network (Hirose et al., 2004; Nakajima et al., 2004) but was identified as a proapoptotic protein of the BH3-only protein family (Boyd et al., 1994; Yasuda and Chinnadurai, 2000). Although both novel SNARE proteins are localized in the ER, Okumura et al. (2006) reported that D12/p31/mUsel associates with the lysosomal SNARE VAMP7 and participates in lysosomal transport. Syntaxin 18 and D12/mUse1 are localized on phagosomal membranes and are required for ER-mediated phagocytosis (Hatsuzawa et al., 2006).

In the present study, we set out to characterize the mammalian homologs of the yeast SNAREs Ufe1, Use1, Sec20, and Sec22 and to investigate their interaction in vivo. We show by FRET that mSec20/ BNIP1 and mUse1/D12 form a SNARE complex with $\mathrm{mSec} 22 \mathrm{~b}$ in the ER of living cells. We demonstrate that the SNARE proteins mSec22b, mSec20/BNIP1 and $\mathrm{mUse} 1 / \mathrm{D} 12$ are present in COPI-coated vesicles and interact with components of the retrograde transport machinery, COPI subunits and the KDEL receptor ERD2.

\section{Materials and methods}

\section{Materials}

Vero cells (ECACC 84113001) were grown in Dulbecco's modified Eagle's medium (DMEM) supplemented 
with $10 \%(\mathrm{v} / \mathrm{v})$ heat inactivated FCS, $100 \mathrm{U} / \mathrm{ml}$ penicillin, $0.1 \mathrm{mg} / \mathrm{ml}$ streptomycin and $2 \mathrm{mM}$-glutamine at $37^{\circ} \mathrm{C}$ and $10 \% \quad \mathrm{CO}_{2}$. Polyclonal antibodies against mSec22b, mUse1/D12, mSec20/BNIP1, ERD2 and CTX-A were obtained as described in the supplementary online material (Table S1). Monoclonal antibodies against rBet1, syntaxin 18 and ERCIC53 were provided by J.C. Hay (University of Michigan), M. Tagaya (School of Life Sciences, Tokyo) and H.P. Hauri (Biozentrum, Basel), respectively. Polyclonal rabbit antibodies against $\beta^{\prime}$-COP and $\delta$-COP came from B. Brügger (Biochemistry Center, Heidelberg).

\section{Fluorescent and non-fluorescent fusion proteins}

Vectors coding for SNARE-GFP variant fusion proteins were constructed using standard PCR and cloning procedures. Deletion and point mutations were created by PCR using appropriate primers (supplementary online material; Table S2).

\section{cDNA transfections of Vero cells}

Confluent Vero cells were trypsinized, washed with PBS, and resuspended in $300 \mu \mathrm{l}$ cytomix $(120 \mathrm{mM} \mathrm{KCl}$, $10 \mathrm{mM} \mathrm{KH}_{2} \mathrm{PO}_{4}, 10 \mathrm{mM} \mathrm{K}_{2} \mathrm{HPO}_{4}, 2 \mathrm{mM}$ EGTA, $5 \mathrm{mM}$ $\mathrm{MgCl}_{2}, 25 \mathrm{mM}$ Hepes, $0.15 \mathrm{mM} \mathrm{CaCl}, 5 \mathrm{mM}$ glutathione, and $2 \mathrm{mM}$ ATP, $\mathrm{pH}$ 7.4) containing the plasmid DNA coding for the indicated proteins. Immediately thereafter cells were transfected by electroporation (Gene Pulser II, Bio-Rad, pulse $0.7 \mathrm{kV}, 50 \mu \mathrm{F}$, 1-2 ms) and grown until the experiment.

\section{Immunoprecipitation}

Microsomal membranes were prepared from liver tissue as previously described (Antonin et al., 2000). For immunoprecipitation, microsomal fractions from rat liver or isolated Golgi-derived vesicles (see below) were solubilized in extraction buffer $(50 \mathrm{mM}$ Tris- $\mathrm{HCl}$, $150 \mathrm{mM} \mathrm{NaCl}$, protease inhibitors, $1 \%$ (v/v) Triton $\mathrm{X}-100)$ at a final protein concentration of about $0.3 \mathrm{mg} / \mathrm{ml}$ and rotated for $90 \mathrm{~min}$ at $4{ }^{\circ} \mathrm{C}$. Insoluble material was removed by centrifugation at $100,000 g_{\text {av }}$ for $60 \mathrm{~min}$. Excess amounts of antibodies against Use1, Sec22b, Sec20 or Bet1 were added. After incubation overnight at $4{ }^{\circ} \mathrm{C}$, protein A-Sepharose (Amersham Pharmacia Biotech) was added in amounts sufficient to bind all IgG quantitatively, followed by incubation for $1 \mathrm{~h}$ at $4{ }^{\circ} \mathrm{C}$. The incubation mix was then spun at $1000 g_{\mathrm{av}}$ for $5 \mathrm{~min}$. The sedimented material was washed six times with $200 \mu \mathrm{l}$ each of extraction buffer followed by solubilization in Laemmli-SDS sample buffer. Protein in the unbound material was precipitated (Wessel and Flügge, 1984) and analyzed by immunoblotting.

\section{Bulk live-cell FRET}

GFP2 (Zimmermann et al., 2002) and VenusYFP (Nagai et al., 2002) were used as donor and acceptor fluorophores, respectively. Vero cells were cotransfected with plasmids coding for GFP2 and VenusYFP fusion proteins and grown in complete DMEM. Ten to twelve hours after transfection, cells (10-cm dish) were collected in $500 \mu \mathrm{l}$ PBS and transferred into a quartz cuvette. Spectra were acquired during GFP2 excitation at $390 \mathrm{~nm}$ and during VenusYFP excitation at $498 \mathrm{~nm}$ using a Cary Varian spectrofluorimeter equipped with the program Eclipse, before and after acceptor bleaching with a 530-nm argon laser.

\section{Single live-cell FRET}

CFP and YFP were used as donor and acceptor fluorophores, respectively. Cells expressing fluorescent ERD2 fusion proteins were collected 5-6h after transfection, as strong overexpression of ERD2 leads to its oligomerization and translocation (Aoe et al., 1997; Majoul et al., 2001). The measurements were performed with the microscope set-up described in the supplementary online material (Table S3). Acceptor bleaching was performed using a small 532-nm lowpower laser, inserted into the optical path of the microscope.

\section{FRET analysis}

The spectra obtained using the program Origin were used to calculate $N_{\text {FRET }}$ with Excel according to the equation

$N_{\mathrm{FRET}}=\left(I_{\mathrm{FRET}}-I_{\mathrm{A}} * y-I_{\mathrm{D}} * z\right) / \sqrt{I_{\mathrm{A}_{\mathrm{bb}}} * I_{\mathrm{D}_{\mathrm{ab}}}}$

where $I_{\mathrm{FRET}}, I_{\mathrm{A}}, I_{\mathrm{D}}$ correspond to the FRET, the acceptor (A) and the donor (D) fluorescence intensities, respectively. $y$ and $z$ correspond to the acceptor and the donor bleed through emissions into the FRET signal, respectively. The equation corrects for the expression levels of fluorescent proteins (adapted from (Xia and Liu, 2001)). $y$ equaled zero as there was no direct excitation of Venus-YFP at $390 \mathrm{~nm}$.

For single cell measurements, the CFP, YFP and FRET signals were measured with the appropriate filter sets. To follow laser-induced acceptor bleaching, a 595-nm dichroic beam splitter and a long-path 570-nm emission filter were used. Images were acquired using Metamorph 6 software (Universal Imaging Corporation, West Chester, PA). The images were corrected for background, pixel shift, and CFP bleed through into the YFP channel using the program Imspector (kindly provided by A. Schönle, Department of 
Nanobiophotonics, this institute). Normalized FRET values ( $N_{\text {FRET }}$ ) were then calculated using Eq. (1). $y$ and $z$ amounted to $4 \%$ and $56 \%$, respectively. For an operational definition of perinuclear $(\mathrm{N})$ and peripheral (P) intracellular regions, the whole cell picture given for the YFP fluorescence was taken as reference. The signal intensity scale was set to saturation limit. The region where the fluorescent signal values exceeded $50 \%$ of the maximal signal was defined as the "perinuclear" region, the values below $50 \%$ as the "peripheral" region.

\section{Bimolecular fluorescence complementation (BiFC)}

Plasmids coding for SNAREs fused to $-\mathrm{YN}$ and $-\mathrm{YC}$ fragments $(\mathrm{N}$ - and $\mathrm{C}$-terminal portions of YFP, respectively) were cotransfected into Vero cells together with pECFP, used to correct for the degree of expression of the fusion proteins. Twenty-four hours after transfection, cells were imaged in PBS at $37^{\circ} \mathrm{C}$ using the microscope set-up and the YFP and CFP filter sets described in the supplementary online material (Table S3). Images were analyzed using Metamorph 6 software. The degree of fluorescence complementation was calculated according to the equation

$R_{\mathrm{BiFC}}=\left(I_{\mathrm{YFP}}-\alpha * I_{\mathrm{CFP}}\right) / I_{\mathrm{CFP}}$

where $\alpha$ corresponds to the CFP bleed through into the YFP channel. The percentage of cells showing fluorescence complementation was plotted against the $R_{\mathrm{BiFC}}$ values using the Sigma Plot program, and variation analysis was performed using the non-parametric method of Mann and Whitney (1947).

\section{Preparation of Golgi stacks and isolated COPI vesicles}

Golgi stacks were prepared from rat livers (Hui et al., 1998). The budding assay was carried out by incubating the isolated Golgi stacks ( $300 \mu \mathrm{g}$ protein) in phosphatebuffered sucrose at $37^{\circ} \mathrm{C}$ for $15 \mathrm{~min}$ under different conditions. The phosphate-buffered sucrose was or was not supplemented with cytosol (Malsam et al., 2005), $100 \mu \mathrm{M}$ GTP $\gamma \mathrm{S}, 5 \mathrm{mM}$ ATP and an ATP-regenerating system or with cytosol, $5 \mathrm{mM}$ GTP, $5 \mathrm{mM}$ ATP and ATP- and GTP-regenerating systems. All samples contained $5 \mathrm{mM}$ magnesium acetate and $100 \mathrm{mM}$ potassium glutamate. Samples were then centrifuged at $15,000 g_{\text {av }}$ for $20 \mathrm{~min}$ to sediment the Golgi membranes. The supernatant was spun for $1 \mathrm{~h}$ at $130,000 g_{\mathrm{av}}$ to sediment the vesicles. The contents of the fractions were analyzed by immunoblotting and immuno-electron microscopy.

\section{Immuno-electron microscopy of ultrathin cryosections}

Golgi membranes and vesicles were fixed with $2 \%$ paraformaldehyde (PFA) in $0.1 \mathrm{M}$ sodium phosphate, $\mathrm{pH} 7.4$, for $16 \mathrm{~h}$ at $4{ }^{\circ} \mathrm{C}$. The pellets were postfixed with $4 \%$ PFA $/ 0.1 \%$ glutaraldehyde for $2 \mathrm{~h}$ on ice. Cryosections were prepared as previously described (Liou et al., 1996; Tokuyasu, 1973; Wenzel et al., 2005), labeled for the indicated antigens and examined with a Philips CM120 electron microscope and a TVIPS CCD camera system.

\section{Immunofluorescence}

Vero cells were fixed in 4\% PFA and subsequently blocked with $50 \mathrm{mM} \mathrm{NH} \mathrm{NH}_{4}$. Cells were permeabilized with $0.1 \%(\mathrm{w} / \mathrm{v})$ saponin $/ 3 \%(\mathrm{w} / \mathrm{v})$ BSA in PBS. The bound first antibody was detected using a 1:1000 dilution of either a Cy3-conjugated goat anti-rabbit or a Cy5-conjugated anti-mouse antibody (Jackson ImmunoResearch Laboratories). Cells were mounted in Dako Medium and analyzed with the set-up described in the supplementary online material (Table S3).

\section{Results}

The SNAREs mSec22b, mUse1/D12, and mSec20/ BNIP1 are distributed between the ER, ERGIC and the Golgi complex

Mammalian homologs for Use1p and Sec20p were identified by database searches and named mammalian Use1 (mUse1/D12 from rat) and mammalian Sec20 (mSec20/BNIP1 from mouse, supplementary online material, Fig. S1), although the homology is relatively low for mSec20/BNIP1. Polyclonal antibodies were raised against the corresponding antigens (supplementary online material, Table S1). After purification by affinity, each antibody showed a single band on Western blots of rat liver crude microsomal preparations (supplementary online material, Fig. S2A).

As a first step to characterize these SNAREs their subcellular localization was determined. Sec22 as the recycling R-SNARE is expected to be present in the ER, the ERGIC as well as in the Golgi. By contrast, Q-SNAREs required for retrograde traffic to the ER do not have to leave the ER to function. Vero cells stained with these antibodies showed widespread punctate and reticular staining patterns resembling those typical of the ER, ERGIC and the Golgi complex but with subtle differences (Fig. 1). In order to differentiate between these compartments, double-labeling experiments were performed with the ER marker calnexin, 

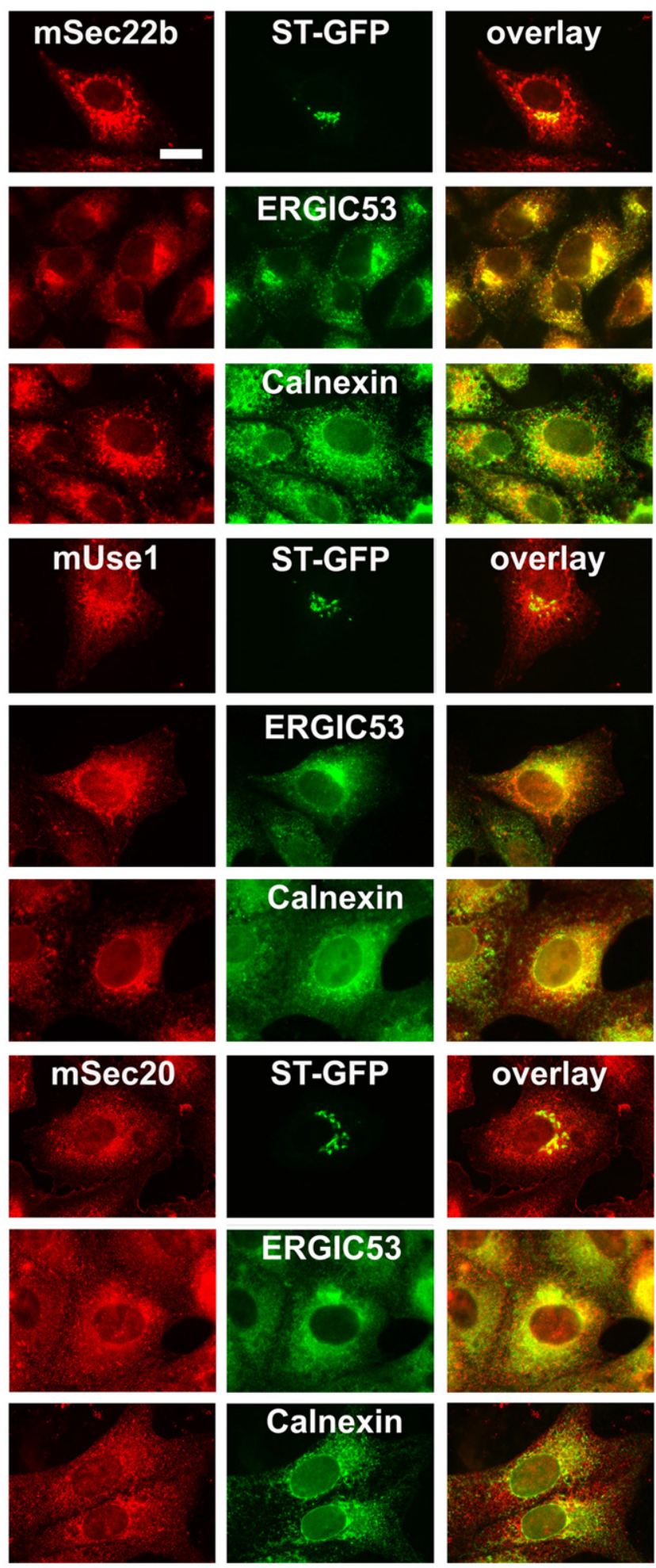

Fig. 1. Localization of retrograde SNARE proteins in Vero cells. Rabbit polyclonal antibodies were used to detect SNAREs, and mouse monoclonal antibodies to detect the ER marker calnexin and the ERGIC marker ERGIC53. The sulfotransferase (ST) was expressed as an EGFP fusion protein to label the Golgi. SNARE proteins are presented in red, the organelle-specific markers in green. Colocalization is indicated in yellow in the overlays. Bar: $20 \mu \mathrm{m}$. the Golgi marker 2-OST (3'-phosphoadenylyl-sulfate: uronyl-2-O-sulfotransferase) and ERGIC53 as marker for the ERGIC. The distribution of $\mathrm{mSec} 22 \mathrm{~b}$ was very similar to that of ERGIC53, and showed significant overlap with 2-OST and with the ER marker calnexin (Fig. 1, top) in agreement with data obtained by immunogold electron microscopy (Hay et al., 1998). mUse1/D12 colocalized only partially with ERGIC53 but showed more overlap with calnexin and also with 2-OST. mSec20/BNIP1 appeared to be present at low concentration in the ER, the ERGIC and the Golgi. Our data indicate that all three SNAREs travel between ER, ERGIC and Golgi. However, they differed in the distribution between these compartments. The main steady-state pool of mSec22b was in the ERGIC, mUse1/D12 was most abundant in the ER. mSec20/ BNIP1 was more evenly distributed between these three compartments.

We also investigated requirements for localization of $\mathrm{mUse} 1 / \mathrm{D} 12$ to the early secretory pathway. A YFPfusion with the transmembrane domain of $\mathrm{mUse} 1 / \mathrm{D} 12$ escaped to the plasma membrane. A YFP-fusion with mUse1/D12 lacking the SNARE motif was found in the ER (supplementary online material, Fig. S4) indicating that the retention of mUse1/D12 required the N-terminal portion upstream of the SNARE motif but not the SNARE motif or the transmembrane domain.

The SNAREs syntaxin 18, mSec22b, mUse1/D12, and $\mathrm{mSec} 20 / \mathrm{BNIP1}$ interact to form a genuine SNARE complex in vivo

As a first approach to investigate whether the SNAREs studied here interact with each other, we performed coimmunoprecipitations from Triton X-100 extracts of rat liver microsomal membranes. As shown in Fig. 2A-C, immunoprecipitation with affinitypurified antibodies against mUse1/D12, mSec22b, or mSec20/BNIP1 precipitated not only the targeted antigens, but also the other three SNARE proteins of the postulated SNARE complex consisting of syntaxin $18, \mathrm{mUse} / \mathrm{D} 12, \mathrm{mSec} 20$ and $\mathrm{mSec} 22 \mathrm{~b}$. The degree of coprecipitation was substantial: not only the precipitated SNARE, but also the coprecipitating partners were visibly depleted from the starting material (particularly in immunoprecipitations carried out with anti-mUse1/D12 and -mSec20 antibodies). This indicates that a large fraction of these SNAREs is present in a SNARE complex at steady state.

No coprecipitation of the anterograde SNARE rBet1 was observed. While our data support the notion that rBet1 does not interact with the retrograde Q-SNAREs, one would expect rBet1 to coprecipitate with mSec22b that operates in both trafficking steps. We therefore carried out additional immunoprecipitations using an 
A
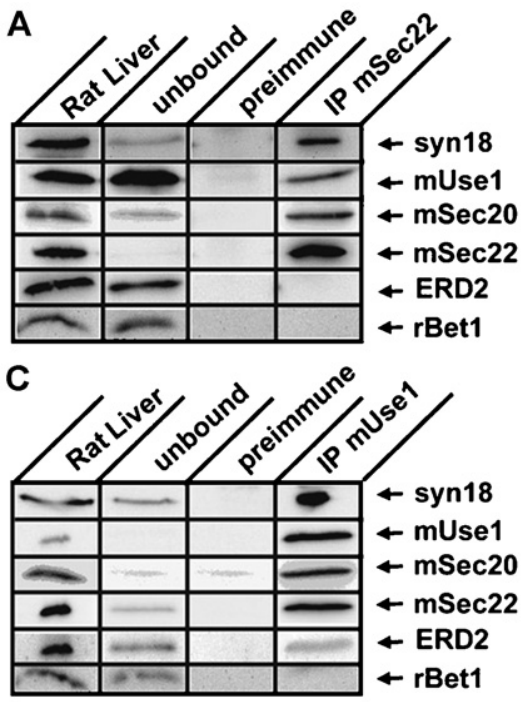

$\mathbf{F}$

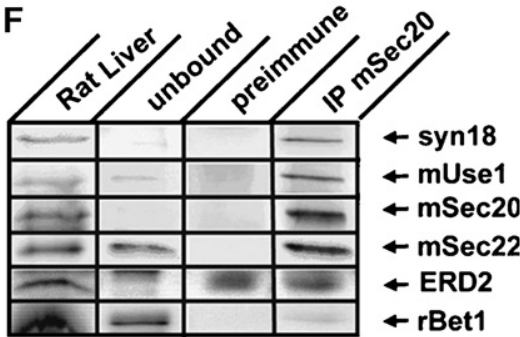

B

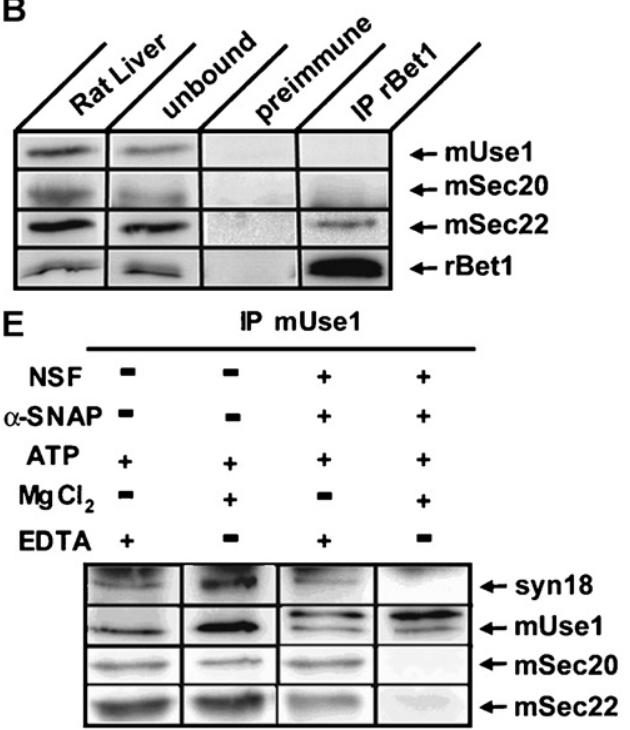

Fig. 2. Coimmunoprecipitation of syntaxin $18, \mathrm{mUse} 1 / \mathrm{D} 12, \mathrm{mSec} 20 / \mathrm{BNIP} 1$, and $\mathrm{mSec} 22 \mathrm{~b}$ indicates the existence of a specific SNARE complex. Detergent extracts from rat liver microsomal membranes were incubated with affinity-purified antibodies against mSec22b (A), mUse1/D12 (B), mSec20/BNIP1 (C), rBet1 (D), or with preimmune serum, followed by precipitation with protein A-Sepharose. Precipitates were analyzed by $12 \%$ SDS-PAGE and immunoblotting. The loaded starting material ("Rat Liver") corresponds to $5 \%$ of the material used for immunoprecipitation. The loaded "unbound" fraction corresponds to $4 \%$ of the total unbound proteins. No unspecific binding to preimmune sera was observed. (E) The SNARE complex extracted from microsomal membranes dissociates in the presence of NSF and $\alpha$-SNAP provided ATP and $\mathrm{Mg}^{2+}$ are present. The membrane extracts were incubated for $1 \mathrm{~h}$ at $16^{\circ} \mathrm{C}$ in the presence $(+)$ or absence $(-)$ of the indicated components: NSF $(50 \mathrm{nM}), \alpha-\mathrm{SNAP}(2 \mu \mathrm{M})$, ATP $(5 \mathrm{mM}), \mathrm{MgCl}_{2}(8 \mathrm{mM})$ and EDTA $(2 \mathrm{mM})$. Note that anti-mUse1/D12 antibodies recognize $\alpha$-SNAP at higher concentrations (i.e. in the presence of added recombinant $\alpha$-SNAP (E), or in the Golgi and vesicle fractions (Fig. 5A)).

anti-rBet1 antibody (Fig. 2D). No significant amounts of the retrograde Q-SNAREs were found in the precipitate, and only a relatively small amount of $\mathrm{mSec} 2 \mathrm{~b}$ was detected, in agreement with the findings of Hay et al. (1997). Considering that Hay et al. (1998) also failed to observe coprecipitation of syntaxin 5 and membrin with $\mathrm{mSec} 22$, it is conceivable that at steady state, $\mathrm{mSec} 22 \mathrm{~b}$ is preferentially associated with the retrograde Q-SNAREs.

To exclude that the association of the retrograde SNAREs detected in the membrane extracts is induced during or after detergent solubilization, trace amounts of $\left[{ }^{35}\right.$ S]methionine-labeled mSec20/BNIP1 were added to the membranes before extraction and immunoprecipitation of mUse1/D12. Only very small amounts of labeled mSec20/BNIP1 were found in the precipitate regardless of whether antibody or preimmune serum was used, whereas specific coprecipitation of mSec20/BNIP1 was as effective as shown above (supplementary online material, Fig. S3). These data confirm that the coprecipitating SNAREs are associated with each other in microsomal membranes.

NSF and $\alpha$-SNAP disassemble SNARE complexes in an ATP-dependent manner (Sollner et al., 1993b). NSF-driven disassembly can therefore be used as a tool to differentiate whether the coprecipitating SNAREs are assembled in a genuine SNARE complex via the SNARE motifs or whether they are associated with each other by other means (e.g. by interaction via their transmembrane domains). We therefore incubated the membrane extracts in the presence of recombinant NSF, $\alpha$-SNAP and ATP prior to immunoprecipitation. As shown in Fig. 2E, no coprecipitation of syntaxin $18, \mathrm{mSec} 20$ or $\mathrm{mSec} 22 \mathrm{~b}$ was observed although mUse1/D12 was effectively precipitated. We conclude that the four SNAREs investigated here are associated with each other in a genuine SNARE complex. 
Next we investigated by FRET whether the proteins associate with each other in living cells. Expression of GFP-tagged mSec22b, mSec20/BNIP1 and mUse1/D12 resulted in an intracellular distribution that was similar to that observed for the corresponding endogenous proteins (supplementary online material, Fig. S2B). In contrast, the distribution of YFP-syntaxin 18 varied between different experiments and did not always coincide with the reticular distribution of its endogenous counterpart. Therefore, this protein was excluded from the FRET analysis.

Under steady-state conditions, FRET measurements of cell suspensions using spectrofluorimetry showed significant FRET between the following pairs: (1) GFP2-mUse1 and VenusYFP-mSec22b, (2) GFP2mUse 1 and VenusYFP-mSec20/BNIP1, and (3) GFP2mSec22 and VenusYFP-mSec20/BNIP1 (Fig. 3A). The $N_{\text {FRET }}$ values obtained for the three SNARE-pairings amounted to about 0.22 (Fig. 3B). These values are significantly higher ( $\sim 7$ fold) than the FRET values obtained in cells coexpressing either soluble GFP2 and VenusYFP, or two membrane GFPvariant fusion proteins residing in different compartments (CD63, residing in lysosomes and a sulfotransferase (2ST) located in the Golgi (Egner et al., 2004), Fig. 3B). A maximum FRET signal (normalized FRET value or $N_{\text {FRET }} 0.36 \pm 0.02$ ) was measured with two fluorescent protein moieties separated by a short spacer (14 amino acids). A second positive control resulting in $N_{\text {FRET }}$ values of about 0.16 was obtained using overexpressed ERD2-VenusYFP and ERD2-GFP2 (Fig. 3B). The KDEL receptor ERD2 oligomerizes depending on the degree of overexpression and its occupancy by KDEL proteins (Aoe et al., 1997; Majoul et al., 2001).

Next, we used FRET in combination with fluorescence microscopy of single living cells to analyze the subcellular localization of the complexes (Fig. 3C). While FRET was observed for the combinations described above, we noted that the normalized FRETvalues measured in the periphery of the cells were consistently higher than in the perinuclear area (Fig. 3D). This could indicate an increased interaction between the SNARE proteins when they are close to or in the ER membrane or in peripheral elements of the ERGIC. To exclude that the observed FRET signals are due to overexpression (membrane crowding) rather than to physiologically relevant protein-protein interactions, we co-expressed CFP-Bet1 with YFP-mUse1. No FRET signal was observed in agreement with the immunoprecipitation experiments. Furthermore, the FRET signal produced by the combination CFP-Bet1/YFP-mSec22b was low (although slightly stronger than that observed for CFP-Bet1/YFP-mUse1). These results are in line with the low levels of anterograde SNARE complexes obtained by coimmunoprecipitation (Fig. 2).
FRET was used to measure homo-oligomerization of SNAREs investigated here in vivo. Coexpression of CFP-mUse1 and YFP-mUse1, or of CFP-mSec20/ BNIP1 and YFP-mSec20/BNIP1 resulted in moderate, but significant FRET signals (Fig. 3D, $N_{\text {FRET }} 0.058 \pm$ 0.016 and $0.043 \pm 0.019$, respectively) indicating some homo-oligomerization. By contrast, there was no indication of homo-oligomerization of $\mathrm{mSec} 22 \mathrm{~b}$ fusion proteins $\left(N_{\text {FRET }} 0.023 \pm 0.015\right)$ compared to coexpression of soluble fluorescent proteins $\left(N_{\text {FRET }} 0.015 \pm\right.$ 0.018 ) or of CFP-Bet1 and YFP-mUse1. The size of the FRET signal was similar in perinuclear and peripheral areas of the cell suggesting that it occurs in the ER as well as in the Golgi in contrast to the larger peripheral FRET signals obtained for SNARE complexes. Deletion of the SNARE motif in mUse1/D12 did not diminish the normalized FRET signal $\left(N_{\text {FRET }}\right.$ $0.054 \pm 0.029)$ indicating that $\mathrm{mUse} 1 / \mathrm{D} 12$ does not homo-oligomerize via its SNARE motif.

To gain an independent line of evidence for interactions occurring between mSec22b, mSec20/BNIP1 and mUse1/D12 in living cells, we applied BiFC. This approach is based on complementation of nonfluorescent fragments of YFP that are able to irreversibly reconstitute the fluorophore when brought together by interactions between proteins fused to each fragment (Hu et al., 2002). When mSec22b linked to the $\mathrm{N}$-terminal YFP fragment (YN-Sec22) was co-expressed with mSec20/BNIP1 linked to the C-terminal YFP fragment (YC-Sec20), reconstituted YFP fluorescence (BiFC fluorescence) was detected in a reticular structure, most likely the ER (Fig. 4A, top panel). The same holds for the coexpressions of YN-mUse1/YC-mSec20/BNIP1 (Fig. 4B, lower panel) and $\mathrm{YN}-\mathrm{mUse} / \mathrm{YC}-\mathrm{mSec} 22 \mathrm{~b}$ (Fig. 4C). Stochastic assembly of YN and YC can be excluded as cells coexpressing the non-fused $\mathrm{N}$ - and C-terminal fragments of YFP showed only negligible YFP fluorescence (data not shown). As an additional control, we co-expressed YN-Sec22 and YN-mUse1, respectively, with $\mathrm{YC}$ fused to a C-terminal fragment of mSec20/BNIP1 that contains the transmembrane domain but lacks most of the cytoplasmic portion including the SNARE motif. Less than $1.3 \%$ of cells showed a fluorescent ratio $\geqslant 0.5$ compared to about $44 \%$ upon expression of intact SNAREs, indicating an almost complete loss of interaction (Fig. 4A). The degree of overexpression and the localization of the mutants were similar to those of WT-SNARE fusion proteins (supplementary online material, Fig. S4B). Fluorescence complementation was strongly diminished when YC-Sec22b was co-expressed with YN fused to an mUse1/D12 variant lacking the SNARE motif (Fig. 4C). Together, our data show that the SNAREs studied here specifically interact with each other in living cells, and furthermore, that this interaction requires the presence of the SNARE motifs. 
It is difficult to predict the position occupied by mUse1/D12 in the SNARE complex because the sequence of mUse1/D12 is very divergent. Specific point mutations can be used to obtain structural information about SNARE complexes. Salt bridges between surfaceexposed residues of the C-terminal SNARE motif of
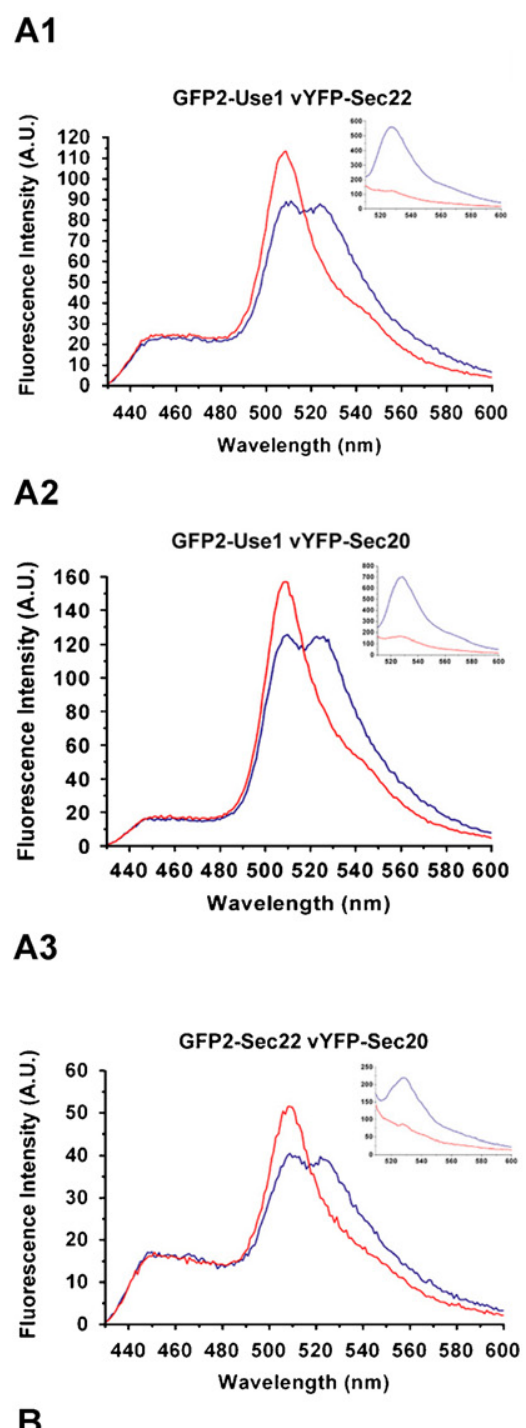

B

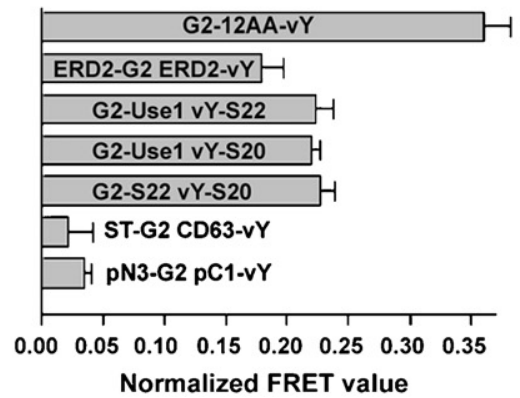

SNAP25 (Qc) and synaptobrevin (Sb2) (SNAP25-R 161 $_{1}$ Sb2-E 41 and SNAP25-D $186 / S b 2-R_{66}$, (Fasshauer et al., 1998)) or of mBet1 (Qc) and $\mathrm{mSec} 22 \mathrm{~b}\left(\mathrm{mBet}^{-\mathrm{K}_{47}}\right.$ $\mathrm{mSec}_{22} \mathrm{~b}-\mathrm{D}_{144}$ and mBet1-D $\mathrm{D}_{72} / \mathrm{mSec}_{2} 2 \mathrm{~b}-\mathrm{K}_{169}$ (Joglekar et al., 2003)) are essential for SNARE complex formation. According to the alignments, $\mathrm{K}_{195}$ and
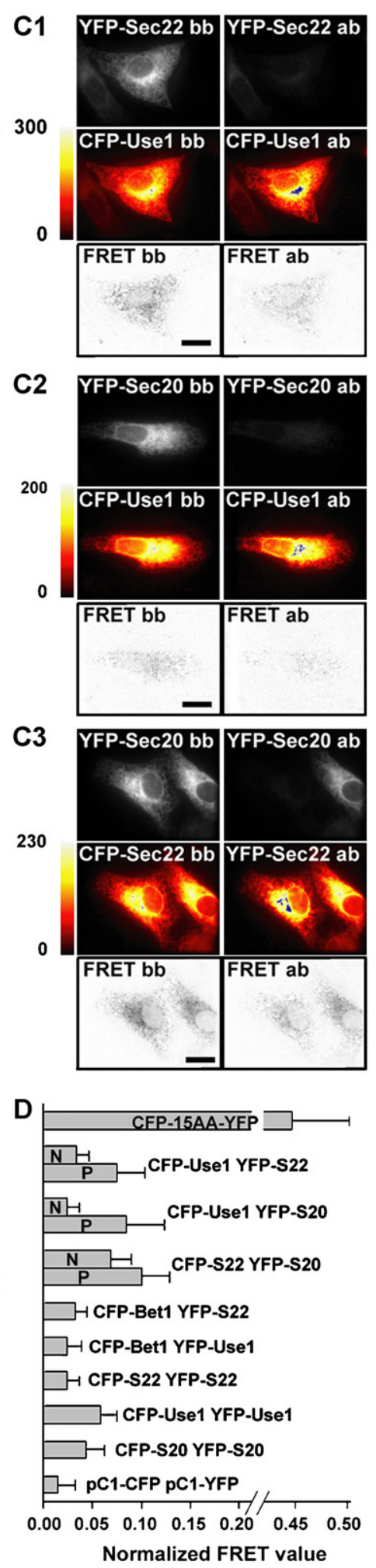
$\mathrm{D}_{220}$ in mUse1/D12 may occupy similar positions as $\mathrm{K}_{47}$ and $\mathrm{D}_{72}$ in mBet1 (supplementary online material, Fig. S4A). We studied by BiFC whether a similar salt bridge formation might be involved in the interaction between $\mathrm{mSec} 22 \mathrm{~b}$ and $\mathrm{mUse} 1 / \mathrm{D} 12$. Coexpression of the $\mathrm{N}$-terminal fragment of YFP fused to a doubly mutated mSec22b (K144D, D169A; Sec22dm-YC) and with YC-WT-mUse1 resulted in a significant left shift in the histogram (Fig. 4D) demonstrating a decreased interaction. The double mutation in the SNARE motif of $\mathrm{mSec} 22 \mathrm{~b}$ had no effect on the steady-state localization (supplementary online material, Fig. S4C) and expression levels. This result indicates that salt bridges are involved in interaction between $\mathrm{mSec} 22 \mathrm{~b}$ and mUse1/D12. Our data suggest that mUse1/D12 is at the same position as Bet1 in the respective SNARE complex and therefore functions as a Qc-SNARE.

\section{Sorting of retrograde SNARE proteins into COPI vesicles and interaction with the KDEL receptor ERD2}

The data presented so far show that the putative retrograde SNAREs mSec22b, mUse1/D12, mSec20/ BNIP1 and syntaxin 18 form a stable SNARE complex in intact cells. While the complex appears to be preferentially localized to peripheral structures (ER, ERGIC), we found all four proteins in the perinuclear area where they colocalize with Golgi markers. Regardless of whether the Golgi pool of these SNAREs is due to cycling (e.g. mSec22b) or due to escape from the ER (Q-SNAREs), they need to be sorted into COPI-coated vesicles in order to return to the ER. Sorting into COPIcoated vesicles would thus provide additional convincing evidence that, unlike in yeast (Burri et al., 2003), not only mSec22 but also the three Q-SNAREs cycle between the ER and the ERGIC or cis-Golgi. To examine this, we used an in vitro assay for the formation of COPI vesicles and tested for the recruitment of SNAREs. COPI vesicles (V2, V3) were generated from purified Golgi stacks (G2, G3, Fig. 5A) and analyzed by Western blotting. Control reactions (G1) were incubated without additions.

mSec20/BNIP1 and syntaxin 18 as well as ERGIC53 were strongly enriched in V2 and V3 COPI vesicle fractions compared to starting Golgi fractions and to the $\mathrm{V} 1$ control fractions. mUse $1 / \mathrm{D} 12$ and $\mathrm{mSec} 22 \mathrm{~b}$ were clearly present in vesicle fractions V2 and V3 in similar concentrations as in Golgi membranes $(100 \mu \mathrm{g}$ of protein loaded for Golgi compared to $50 \mu \mathrm{g}$ for vesicles). Vesicular fractions contained COPI as indicated by probing for $\beta^{\prime}$-COP. These fractions did not consist of fragmented Golgi because they are enriched in the ERGIC marker ERGIC53.

The presence of $\mathrm{mSec} 22 \mathrm{~b}, \mathrm{mUse} 1 / \mathrm{D} 12$ and $\mathrm{mSec} 20 /$ BNIP1 on COPI vesicles was confirmed by immunoelectron microscopy and double labeling (Fig. 5C). The mSec20/BNIP1 staining (Fig. 5C a-c) was weaker than that of $\mathrm{mSec} 22 \mathrm{~b}$ and $\mathrm{mUse} / \mathrm{D} 12$, which is in line with the immunoblotting experiments (Fig. 5A). Importantly, $\mathrm{mSec} 22 \mathrm{~b}$, mUse1/D12, and mSec20/BNIP1 were occasionally found in various combinations on the same vesicles (Fig. $5 \mathrm{C} \mathrm{g-i}$ ), which could be identified as COPI vesicles as they costained for SNAREs and $\beta^{\prime}$-COP. We could also observe colocalization of ERD2 and retrograde SNAREs on the same vesicle (Fig. $5 \mathrm{C} \mathrm{d}-\mathrm{f}$ ).

As we observed less SNARE complexes in the Golgi area we tested by coimmunoprecipitation using detergent extracts from V2 and V3 vesicles whether SNAREs are found in a complex in COPI vesicles. Only minor amounts of $\mathrm{mSec} 22$ and $\mathrm{mUse} 1 / \mathrm{D} 12$ were

Fig. 3. Pairwise interactions between retrograde SNAREs in living cells as measured by FRET. The measurements were performed by spectrofluorimetry (A1-A3; B) or by single cell fluorescence microscopy (C1-C3; D). Suspensions of cells coexpressing GFP2mUse1 and vYFP-mSec22b (A1), GFP2-mUse1 and vYFP-mSec20/BNIP1 (A2), or GFP2-mSec22b and vYFP-mSec20/BNIP1 (A3) were excited at $390 \mathrm{~nm}$ and subsequently at $498 \mathrm{~nm}$ (insets). Emission spectra were acquired before (blue line) and after (red line) acceptor photobleaching. (B) Normalized FRET values obtained for the different SNARE pairs. Cells expressing either the G2-12AA-vY control construct (GFP2 and vYFP separated by a 12-amino-acid linker) or the ERD2-vYFP/ERD2-GFP2 pair served as positive controls. Cells which coexpressed either the Golgi marker 2-OST-GFP2 (ST-G2) and the lysosomal marker CD63-vYFP, or the free soluble fluorescent proteins GFP2 and vYFP (pN3-G2/pC1-vYFP) served as negative controls. Shown are mean values \pm S.D. of at least five independent experiments in each group. (C) Vero cells coexpressing CFP-mUse1 and YFP-mSec22b (C1), CFP-mUse1 and YFP-mSec20/BNIP1 (C2), or CFP-mSec22b and YFP-mSec20/BNIP1 (C3) were grown on coverslips. Images were acquired in the YFP channel (top rows), in the CFP channel (middle rows), and in the FRET channel (bottom rows) before (bb, left columns) and after (ab, right columns) acceptor photobleaching. Donor fluorescence intensities which exceed after acceptor photobleaching the maximum intensities obtained before bleaching (false color scales on the left) are indicated in blue. (D) Normalized FRET values obtained for the hetero-SNARE pairs ((C1-C3), CFP-Bet1/YFP-mSec22b and CFP-Bet1/YFPmUse1) and the homo-SNARE pairs (CFP-mSec22b/YFP-mSec22b, CFP-mUse1/YFP-mUse1, and CFP-mSec20/YFP-mSec20). Cells expressing CFP-15AA-YFP (CFP and YFP separated by a 15-amino-acid linker) or coexpressing soluble CFP and YFP (pC1-CFP/pC1-YFP) served as controls. Note that for the hetero-SNARE pairs FRET intensity differed strongly between the perinuclear $(\mathrm{N})$ region and the periphery $(\mathrm{P})$. Shown are mean values \pm S.D. from three independent experiments, analyzing for each at least 10 different cells. Bar, $20 \mu \mathrm{m}$. 
coimmunoprecipitated by antibodies against mUse1/ D12 (Fig. 6A) or mSec22 (Fig. 6B), respectively. This indicates that less $\mathrm{mSec} 22$ and $\mathrm{mUse} / \mathrm{D} 12$ are found in SNARE complexes in COPI vesicles than in the cell in general (Fig. 2A and B).
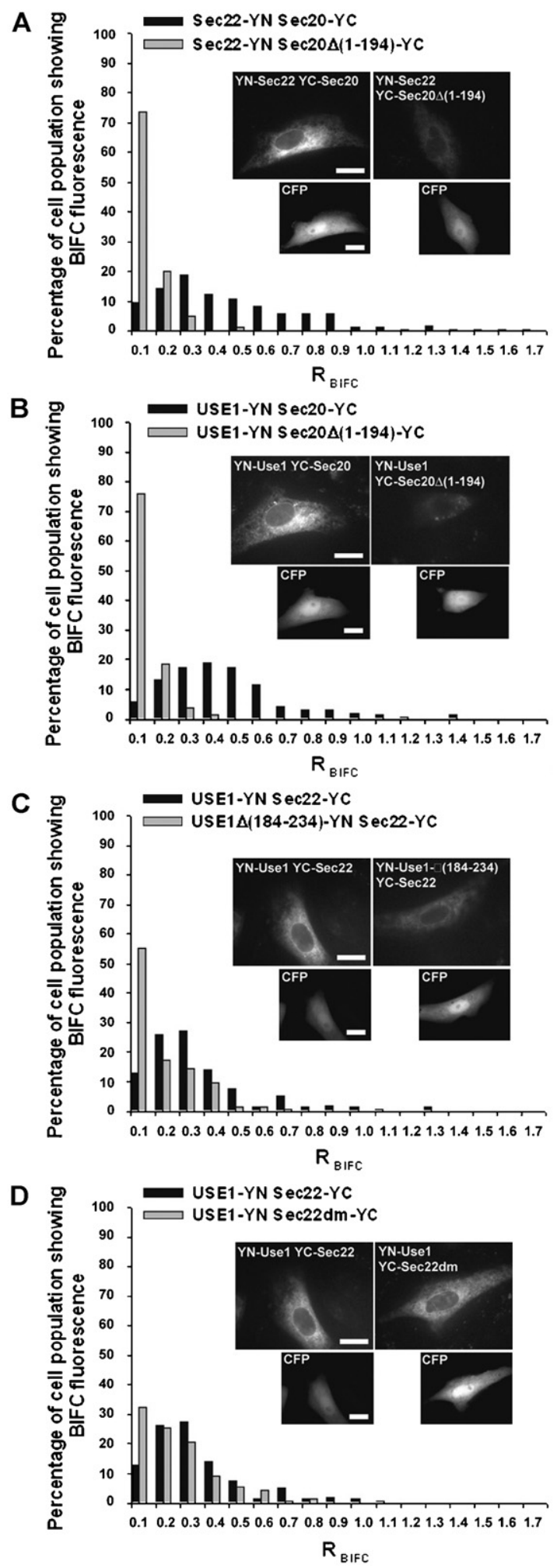

Since COPII complexes bind ER-to-Golgi anterograde SNAREs (Liu et al., 2004; Miller et al., 2002; Mossessova et al., 2003) and COPI complexes bind yeast Bet $1 p$ and Sec22p depending on activated Arf1 $p$ in vitro (Rein et al., 2002; Springer and Schekman, 1998), we tested whether mSec22b and mUse1/D12 interact with subunits of the COPI coat by coimmunoprecipitation using detergent extracts from V2 and V3 vesicles (Fig. 6). $\delta$-COP was coimmunoprecipitated with both anti-mUse1/D12 and anti-mSec22b antibodies (Fig. 6A, B). A significant amount of $\beta^{\prime}$-COP was coprecipitated with anti-mUse1/D12 antibodies (Fig. 6A) whereas no detectable amounts of $\beta^{\prime}$-COP were coimmunoprecipitated with anti-mSec22b antibodies (Fig. 6B). As COP complexes are stable in vivo (Kreis et al., 1995) we may have problems with stability or detection of $\beta^{\prime}$-COP after immunoprecipitation of $\mathrm{mSec} 22 \mathrm{~b}$. Preimmune serum did not precipitate $\delta$-COP or $\beta^{\prime}$-COP indicating that these interactions were specific (Fig. 6C).

COPII coat proteins interact with anterograde SNAREs as well as with cargo in anterograde traffic from ER to the Golgi (Kuehn et al., 1998). Therefore, we have examined whether components of the putative retrograde SNARE complex might interact with a retrograde cargo protein, the KDEL receptor ERD2. Antibodies directed against mUse1/D12 coprecipitated ERD2 from detergent extracts of rat liver microsomal membranes (Fig. 2A). ERD2 was not coimmunoprecipitated with antibodies directed against $\mathrm{mSec} 22 \mathrm{~b}$. This may be due to technical reasons such as sterical hindrance. $\mathrm{mSec} 20$ preimmune serum strongly recognized a band of the same mobility as ERD2 preventing an interpretation of the mSec20-ERD2 coimmunoprecipitation (Fig. 2C). ERD2-YFP and one CFP fusion with a retrograde SNARE protein (CFP-mSec22b, CFP-mUse1 or CFP-mSec20/BNIP1) were co-expressed as an independent second assay and to analyze these

Fig. 4. BiFC shows interactions between retrograde SNAREs in living cells. Vero cells coexpressing the indicated pairs of SNARE-YFP fragment fusion proteins and CFP (reference for the protein expression level) were imaged. Fluorescence indicates complementation of the $\mathrm{N}$ - and C-terminal fragments of YFP. The percentage of the analyzed cell population showing reconstituted YFP fluorescence is plotted against the $R_{\mathrm{BiFC}}$ values. Black bars = cells expressing pairs of WT-SNARE fusion proteins, grey bars cells coexpressing a WT-SNARE fusion protein together with a mutant SNARE fusion protein. (A) and (B) YC-Sec20 $\Delta$ (1-194) $=m$ Sec20/BNIP1 with the N-terminal 194 amino acids deleted and fused to the C-terminal fragment of YFP; (C) YN-Use1 $\Delta(184-234)=$ mUsel/D12 with deleted SNARE motif fused to the N-terminal fragment of YFP; (D) YC-Sec22dm $=m$ Sec $22 b$ with two point mutations (D169A, K144D) fused to the C-terminal fragment of YFP. The differences between wildtype and mutant SNAREs are all statistically significant $(p<0.0001)$. Bars: $20 \mu \mathrm{m}$. 

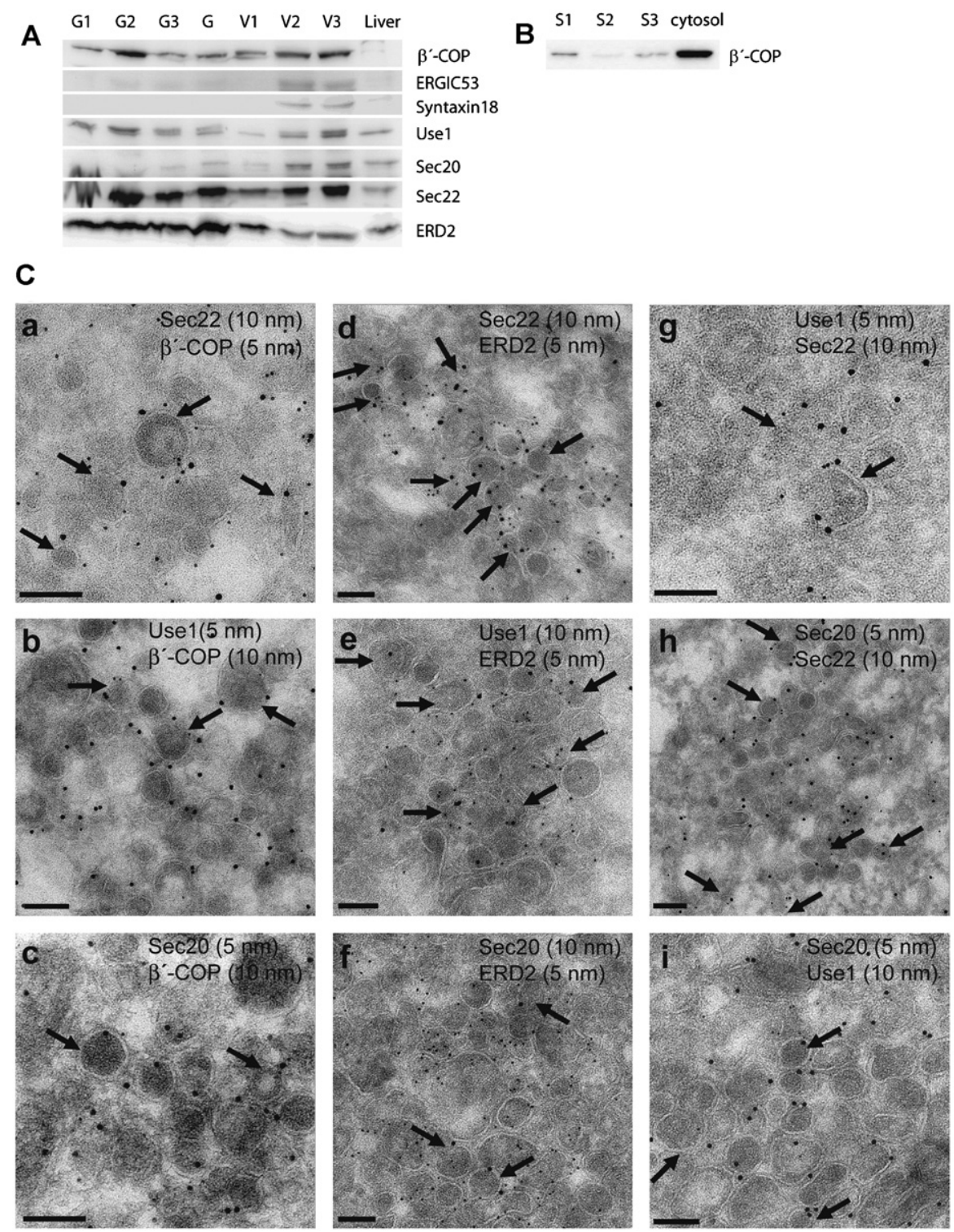

Fig. 5. Vesicles budding from isolated Golgi stacks in vitro contain retrograde SNARE proteins. (A) Golgi stacks were incubated without additives $\left(G_{1}, V_{1}\right)$, in the presence of cytosol, ATP, an ATP-regenerating system, and GTP- $\gamma$-S $\left(G_{2}, V_{2}\right)$, or in the presence of cytosol, ATP, GTP and ATP-GTP-regenerating systems $\left(G_{3}, V_{3}\right) . \mathrm{G}=$ non-incubated Golgi. After incubation, Golgi stacks and vesicles were separated by centrifugation and analyzed for the presence of the indicated proteins by $15 \%$ SDS-PAGE and immunoblotting using $100 \mu \mathrm{g}$ protein for the Golgi and $50 \mu \mathrm{g}$ for the vesicle fractions. (B) The cytosol used for Golgi incubation and the supernatants obtained after isolation of vesicles (S1, S2, S3) were analyzed for the presence of cytosolic $\beta^{\prime}$-COP protein. (C) Vesicles V2 were analyzed by cryo-immuno electron microscopy for the indicated pairs of proteins: $\beta^{\prime}$-COP subunit with (a) mSec22b, (b) mUse1/D12 or (c) mSec20/BNIP1; ERD2 with (d) mSec22b, (e) mUse1/D12 or (f) mSec20/BNIP1; and the SNARE pairs (g) $\mathrm{mUse} 1 / \mathrm{mSec} 22 \mathrm{~b}$, (h) $\mathrm{mSec} 20 / \mathrm{mSec} 22 \mathrm{~b}$ and (i) $\mathrm{mSec} 20 / \mathrm{mUse}$. The size of the gold grains is indicated. Bars: $100 \mathrm{~nm}$.

interactions in single living cells. A moderate but significant, FRET signal could be detected in the peripheral parts of the cells expressing ERD2-YFP and any of the three CFP-SNARE fusions (Fig. 7A and B). In the perinuclear area, FRET signals did not exceed control levels observed for coexpression of ERD2-YFP with the cis-Golgi membrane protein sulfotransferase (ST-CFP) or of soluble fluorescent proteins (Fig. 7B). This indicates that the FRET signal observed between
ERD2-YFP and retrograde SNAREs in the peripheral part of the cell is specific and not due to membrane crowding.

\section{Discussion}

We have shown here that mSec22b, mUse1/D12, mSec20/BNIP1, and syntaxin 18 form a SNARE 

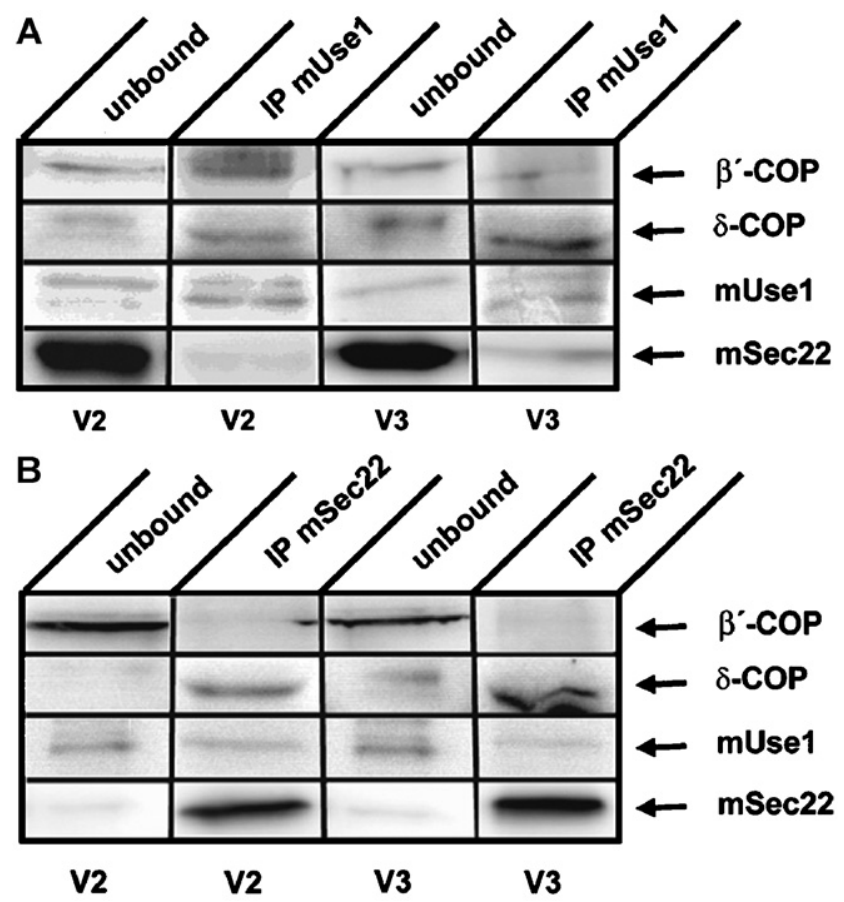

C

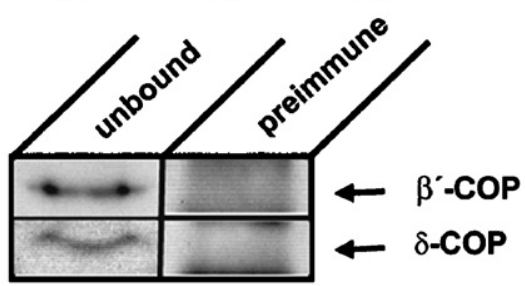

Fig. 6. $m U$ se $1 / D 12$ and $m S e c 22 b$ interact with coatomer subunits. Affinity-purified antibodies against mUse1/D12 (A) or $\mathrm{mSec} 22 \mathrm{~b}$ (B) were used for coimmunoprecipitation of detergent extracts from the $V_{2^{-}}$or $V_{3}$-vesicles fractions. Precipitates were analyzed by $12 \%$ SDS-PAGE and immunoblotting using antibodies against $\beta^{\prime}$-COP, $\delta$-COP, mUse1/D12, or $\mathrm{mSec} 22 \mathrm{~b}$. (C) No unspecific binding to preimmune sera was observed.

complex in living cells with three different techniques, FRET, BiFC and coimmunoprecipitation. Coimmunoprecipitation was performed under condition in which complex formation occurred before solubilization of membranes. Surprisingly, a large fraction of $\mathrm{mSec} 22 \mathrm{~b}$, $\mathrm{mUse} 1 / \mathrm{D} 12, \mathrm{mSec} 20 / \mathrm{BNIP1}$, and syntaxin 18 resided in complexes. These complexes were genuine SNARE complexes assembled via the SNARE motifs due to their sensitivity to NSF and $\alpha$-SNAP. By contrast, only $5-10 \%$ of other SNAREs are found in SNARE complexes (Antonin et al., 2000; Hay et al., 1998; Sollner et al., 1993a). Such stable SNARE complexes are obtained after fusion when all SNAREs are found in the same membrane (cis-SNARE complexes). They are thought to represent an inactive state and have to be dissociated by NSF and $\alpha$-SNAP for a further round of fusion (Jahn et al., 2003). The SNARE complex consisting of $\mathrm{mSec} 22 \mathrm{~b}, \mathrm{mUse} / \mathrm{D} 12, \mathrm{mSec} 20 / \mathrm{BNIP} 1$,
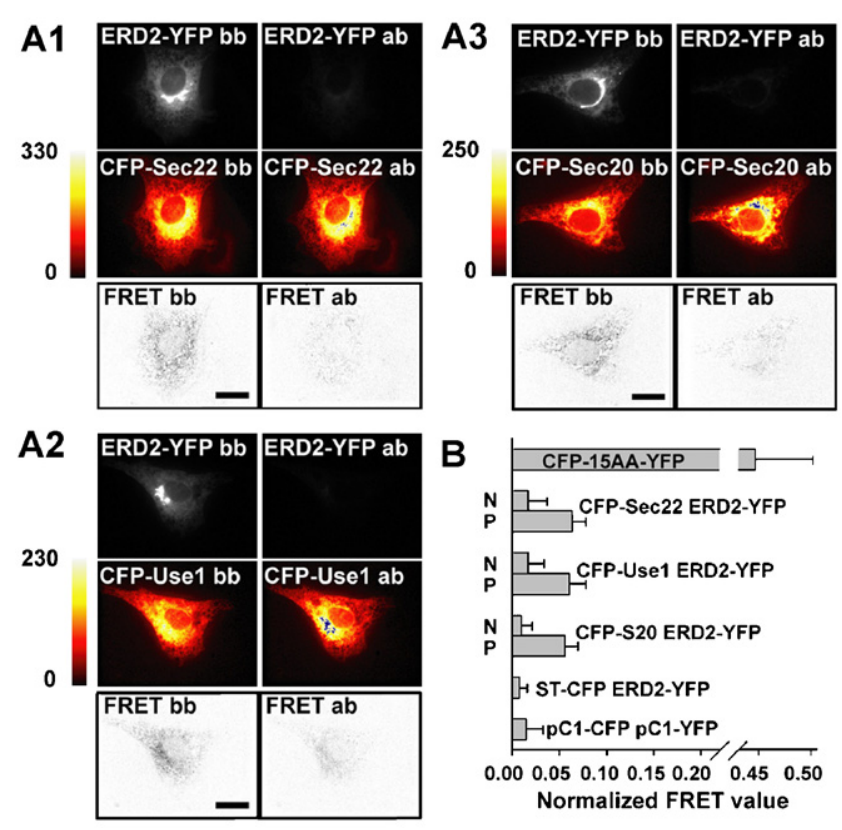

Fig. 7. The putative retrograde SNAREs interact with ERD2, a cargo component of the retrograde Golgi-to-ER transport. (A) FRET measurements in single cells coexpressing ERD2YFP and different retrograde SNARE proteins fused to CFP were performed as in Fig. 3C. Cells coexpressing ERD2-YFP and either CFP-Sec22 (A1), or CFP-Use1 (A2) or CFP-Sec20 (A3) were grown on coverslips. Images were acquired in the YFP channel (top row), in the CFP channel (middle row) and in the FRET channel (bottom row), before (bb, left column) and after (ab, right column) acceptor photobleaching. Donor fluorescence intensities which exceed after acceptor photobleaching the maximum intensities obtained before bleaching (false color scales on the left) are indicated in blue. (B) Shown are mean values \pm S.D. of normalized FRET values for the indicated protein pairs, for the positive control (CFP-15AAYFP) and for the negative controls (ST-CFP/ERD2-YFP and $\mathrm{pC} 1-\mathrm{CFP} / \mathrm{pC} 1-\mathrm{YFP})$. Note that the FRET signal reflecting the interaction between ERD2 and each of the SNARE proteins was mainly detected in the periphery of the cells (P) but was almost absent in the perinuclear region (N). For each condition at least three independent experiments were performed, each analyzing at least 10 cells. The $N_{\text {FRET }}$ values obtained in the periphery of the cells are all statistically significant $\left(p<5 \times 10^{-9}\right)$. Bar: $20 \mu \mathrm{m}$.

and syntaxin 18 was more abundant in peripheral parts of the cells consistent with ER and peripheral parts of the ERGIC as seen by FRET. By contrast, only minor amounts of $\mathrm{mSec} 22 \mathrm{~b}$ could be coprecipitated together with mUse1/D12 using COPI vesicles as starting material, even though immuno-electron microscopy proved that these SNAREs coexist in the same COPI vesicle. This might indicate that a partial or complete SNARE complex in which $m$ Sec $22 \mathrm{~b}$ and $\mathrm{mUse} 1 / \mathrm{D} 12$ coexist does not yet exist at the transport step starting from the Golgi. As uncomplexed SNAREs are required 
for fusion while SNARE complexes are found on target membranes these data suggest that this SNARE complex functions in fusion of COPI vesicles with the ER and/or ERGIC. mSec22 would be the active R-SNARE in COPI vesicles whereas the other SNAREs are cargo. A role of this SNARE complex in retrograde transport is consistent with the established function of the yeast homologs Ufe1p, Sec20p and Use1p in retrograde traffic from the Golgi to the ER (Ballensiefen et al., 1998; Burri et al., 2003; Dilcher et al., 2003; Lewis et al., 1997).

The models for retrograde transport from the Golgi to the ER in mammalian cells are still under discussion. Initially it was thought that only a direct transport from the Golgi to the ER occurs via COPI vesicles. However, recently it has been shown that retrograde traffic can pass through the ERGIC (Appenzeller-Herzog and Hauri, 2006; Ben-Tekaya et al., 2005; Marra et al., 2001; Volpicelli-Daley et al., 2005). Furthermore, an additional retrograde pathway from the cis-Golgi to the ER has been described which seems to be COPIindependent (Girod et al., 1999). It is difficult to determine, whether a SNARE complex functions in anterograde or retrograde traffic between two compartments. Inhibition of one transport step can affect other pathways indirectly. Nakajima et al. (2004) described that overexpression of mSec20/BNIP1 aggregated ER structure but did not influence anterograde transport to the Golgi. Overexpression of a mutant form of syntaxin 18 inhibited anterograde transport to the Golgi (Hatsuzawa et al., 2000), which could be indirect due to inhibition of retrograde transport. Syntaxin 18 is required for cell cycle-dependent reformation of the ER network (Kano et al., 2005), which is consistent with homotypic ER fusion and retrograde traffic to the ER but not with anterograde traffic to the Golgi. Knockdown of mUse1/D12 induced apoptosis in addition to generation of lipofuscin (Okumura et al., 2006) but no defects in anterograde transport of VSV G protein and KDEL receptor. KDEL receptor was still localized at the Golgi consistent with a block of retrograde traffic to the ER. A lysosomal protease accumulated in a Golgi or post-Golgi compartment. However, it is not certain that this is a direct effect. Taken together, these data suggest that the complex consisting of syntaxin $18, \operatorname{mSec} 22 \mathrm{~b}$, $\mathrm{mUse} 1 / \mathrm{D} 12$ and $\mathrm{mSec} 20 / \mathrm{BNIP} 1$ functions in retrograde traffic from the Golgi to the ER.

mUse1/D12 and mSec20/BNIP1 were found to homooligomerize to some degree in vivo by FRET. This homo-oligomerization was independent of the SNARE motif of mUse1/D12. SNARE homo-oligomerization has been suggested to function in exocytosis of synaptic vesicles by forming a ring of SNARE complexes mediated via transmembrane domains (Laage et al., 2000; Ungermann and Langosch, 2005). Clustering of SNAREs in vivo can also involve cholesterol as well as the SNARE motif as shown for syntaxin 1 (Lang et al., 2001; Sieber et al., 2007).

We analyzed COPI vesicles formed from isolated Golgi stacks to follow trafficking of the SNAREs studied here. During budding, mSec22b, mUse1/D12, $\mathrm{mSec} 20 / \mathrm{BNIP} 1$, and syntaxin 18 are sorted into COPI vesicles. mUse1/D12 and COPI subunits could be coimmunoprecipitated. Interaction between SNAREs and vesicle coats is an important mechanism for SNARE localization and possibly for vesicle formation. Interactions between COPII and SNARE proteins have been shown to occur in yeast (Liu et al., 2004; Miller et al., 2002; Mossessova et al., 2003). Betlp and to a lesser extent Sec22p bind Arf1p and COPI after interaction with ARF-GAP in vitro (Rein et al., 2002; Springer and Schekman, 1998). Budding of COPI vesicles from the cis-Golgi requires the interaction of coatomer subunits with Arf1 (Bremser et al., 1999; Sohn et al., 1996). Moreover, budding of retrogradely directed COPI vesicles is also accompanied by a direct interaction of the occupied KDEL receptor ERD2 with COPI and the Arf1-GTPase-activating protein (Arf-GAP, Majoul et al., 2001). Here, we completed the picture further by showing an interaction between ERD2 and retrograde SNARE proteins. However, we cannot distinguish between direct and indirect interactions between these SNAREs and ERD2 or COPI. Our failure to co-immunoprecipitate ERD2 with mSec22b while a FRET signal was observed may be due either to technical reasons or to binding via an intermediate protein. Taken together these data suggest that the budding of retrograde COPI vesicles requires the formation of a large complex, which also includes retrograde SNARE proteins.

\section{Acknowledgments}

We thank Alexander Goroshkov for invaluable help during the set-up of the microscope for live-cell FRET measurements. We are grateful to Beate Veith for excellent technical assistance and Andreas Schönle for help with his image analysis software Imspector. We thank Hans-Dieter Schmitt, Silvio Rizzoli, Dirk Fasshauer, and Reinhard Jahn for helpful comments on the manuscript. This work was supported by a grant from the Deutsche Forschungsgemeinschaft (So 43/63) given to H.-D. Söling.

\section{Appendix A. Supporting Information}

Supplementary data associated with this article can be found in the online version at doi:10.1016/j.ejcb. 2008.07.003. 


\section{References}

Altan-Bonnet, N., Sougrat, R., Lippincott-Schwartz, J., 2004. Molecular basis for Golgi maintenance and biogenesis. Curr. Opin. Cell Biol. 16, 364-372.

Antonin, W., Holroyd, C., Fasshauer, D., Pabst, S., Von Mollard, G.F., Jahn, R., 2000. A SNARE complex mediating fusion of late endosomes defines conserved properties of SNARE structure and function. EMBO J. 19, 6453-6464.

Antonin, W., Fasshauer, D., Becker, S., Jahn, R., Schneider, T.R., 2002. Crystal structure of the endosomal SNARE complex reveals common structural principles of all SNAREs. Nat. Struct. Biol. 9, 107-111.

Aoe, T., Cukierman, E., Lee, A., Cassel, D., Peters, P.J., Hsu, V.W., 1997. The KDEL receptor, ERD2, regulates intracellular traffic by recruiting a GTPase-activating protein for ARF1. EMBO J. 16, 7305-7316.

Aoe, T., Lee, A.J., van Donselaar, E., Peters, P.J., Hsu, V.W., 1998. Modulation of intracellular transport by transported proteins: insight from regulation of COPI-mediated transport. Proc. Natl. Acad. Sci. USA 95, 1624-1629.

Appenzeller-Herzog, C., Hauri, H.P., 2006. The ER-Golgi intermediate compartment (ERGIC): in search of its identity and function. J. Cell Sci. 119, 2173-2183.

Ballensiefen, W., Ossipov, D., Schmitt, H., 1998. Recycling of the yeast v-SNARE Sec22p involves COPI-proteins and the ER transmembrane proteins Ufe1p and Sec20p. J. Cell Sci. 111, 1507-1520.

Ben-Tekaya, H., Miura, K., Pepperkok, R., Hauri, H.P., 2005. Live imaging of bidirectional traffic from the ERGIC. J. Cell Sci. 118, 357-367.

Bennett, M.K., Scheller, R.H., 1993. The molecular machinery for secretion is conserved from yeast to neurons. Proc. Natl. Acad. Sci. USA 90, 2559-2563.

Boyd, J.M., Malstrom, S., Subramanian, T., Venkatesh, L.K., Schaeper, U., Elangovan, B., D'Sa-Eipper, C., Chinnadurai, G., 1994. Adenovirus E1B $19 \mathrm{kDa}$ and Bcl-2 proteins interact with a common set of cellular proteins. Cell 79, 341-351.

Bremser, M., Nickel, W., Schweikert, M., Ravazzola, M., Amherdt, M., Hughes, C.A., Sollner, T.H., Rothman, J.E., Wieland, F.T., 1999. Coupling of coat assembly and vesicle budding to packaging of putative cargo receptors. Cell 96, 495-506.

Burri, L., Varlamov, O., Doege, C.A., Hofmann, K., Beilharz, T., Rothman, J.E., Sollner, T.H., Lithgow, T., 2003. A SNARE required for retrograde transport to the endoplasmic reticulum. Proc. Natl. Acad. Sci. USA 100, 9873-9877.

Chen, Y.A., Scheller, R.H., 2001. SNARE-mediated membrane fusion. Nat. Rev. Mol. Cell Biol. 2, 98-106.

Cosson, P., Lefkir, Y., Demolliere, C., Letourneur, F., 1998. New COP1-binding motifs involved in ER retrieval. EMBO J. 17, 6863-6870.

Dilcher, M., Veith, B., Chidambaram, S., Hartmann, E., Schmitt, H.D., von Mollard, G.F., 2003. Uselp is a yeast SNARE protein required for retrograde traffic to the ER. EMBO J. 22, 3664-3674.

Egner, A., Verrier, S., Goroshkov, A., Söling, H.D., Hell, S.W., 2004. 4Pi-microscopy of the Golgi apparatus in live mammalian cells. J. Struct. Biol. 147, 70-76.
Fasshauer, D., Sutton, R.B., Brünger, A.T., Jahn, R., 1998. Conserved structural features of the synaptic fusion complex: SNARE proteins reclassified as Qand R-SNAREs. Proc. Natl. Acad. Sci. USA 95, 15781-15786.

Girod, A., Storrie, B., Simpson, J.C., Johannes, L., Goud, B., Roberts, L.M., Lord, J.M., Nilsson, T., Pepperkok, R., 1999. Evidence for a COP-I-independent transport route from the Golgi complex to the endoplasmic reticulum. Nat. Cell Biol. 1, 423-430.

Hara-Kuge, S., Kuge, O., Orci, L., Amherdt, M., Ravazzola, M., Wieland, F.T., Rothman, J.E., 1994. En bloc incorporation of coatomer subunits during the assembly of COP-coated vesicles. J. Cell Biol. 124, 883-892.

Hardwick, K.G., Pelham, H.R., 1992. SED5 encodes a 39-kD integral membrane protein required for vesicular transport between the ER and the Golgi complex. J. Cell Biol. 119, 513-521.

Hatsuzawa, K., Hirose, H., Tani, K., Yamamoto, A., Scheller, R.H., Tagaya, M., 2000. Syntaxin 18, a SNAP receptor that functions in the endoplasmic reticulum, intermediate compartment, and cis-Golgi vesicle trafficking. J. Biol. Chem. 275, 13713-13720.

Hatsuzawa, K., Tamura, T., Hashimoto, H., Hashimoto, H., Yokoya, S., Miura, M., Nagaya, H., Wada, I., 2006. Involvement of syntaxin 18, an endoplasmic reticulum (ER)-localized SNARE protein, in ER-mediated phagocytosis. Mol. Biol. Cell 17, 3964-3977.

Hay, J.C., 2001. SNARE complex structure and function. Exp. Cell Res. 271, 10-21.

Hay, J., Chao, D., Kuo, C., Scheller, R., 1997. Protein interactions regulating vesicle transport between the endoplasmic reticulum and Golgi apparatus in mammalian cells. Cell 89, 149-158.

Hay, J.C., Klumperman, J., Oorschot, V., Steegmaier, M., Kuo, C.S., Scheller, R.H., 1998. Localization, dynamics, and protein interactions reveal distinct roles for ER and Golgi SNAREs. J. Cell Biol. 141, 1489-1502.

Hirose, H., Arasaki, K., Dohmae, N., Takio, K., Hatsuzawa, K., Nagahama, M., Tani, K., Yamamoto, A., Tohyama, M., Tagaya, M., 2004. Implication of ZW10 in membrane trafficking between the endoplasmic reticulum and Golgi. EMBO J. 23, 1267-1278.

Hu, C.D., Chinenov, Y., Kerppola, T.K., 2002. Visualization of interactions among bZIP and Rel family proteins in living cells using bimolecular fluorescence complementation. Mol. Cell 9, 789-798.

Hui, N., Nakamura, N., Slusarewicz, P., Warren, G., 1998. Purification of rat liver Golgi stacks. In: Celis, J.E. (Ed.), Cell Biology: A Laboratory Handbook, Vol. 2. Academic Press, San Diego, USA, pp. 46-55.

Jahn, R., Lang, T., Südhof, T., 2003. Membrane fusion. Cell 112, 519-533.

Joglekar, A.P., Xu, D., Rigotti, D.J., Fairman, R., Hay, J.C., 2003. The SNARE motif contributes to rbet 1 intracellular targeting and dynamics independently of SNARE interactions. J. Biol. Chem. 278, 14121-14133.

Kano, F., Kondo, H., Yamamoto, A., Kaneko, Y., Uchiyama, K., Hosokawa, N., Nagata, K., Murata, M., 2005. NSF/ SNAPs and p97/p47/VCIP135 are sequentially required for 
cell cycle-dependent reformation of the ER network. Genes Cells 10, 989-999.

Kreis, T.E., Lowe, M., Pepperkok, R., 1995. COPs regulating membrane traffic. Annu. Rev. Cell Dev. Biol. 11, 677-706.

Kuehn, M.J., Herrmann, J.M., Schekman, R., 1998. COPIIcargo interactions direct protein sorting into ER-derived transport vesicles. Nature 391, 187-190.

Laage, R., Rohde, J., Brosig, B., Langosch, D., 2000. A conserved membrane-spanning amino acid motif drives homomeric and supports heteromeric assembly of presynaptic SNARE proteins. J. Biol. Chem. 275, 17481-17487.

Lang, T., Bruns, D., Wenzel, D., Riedel, D., Holroyd, P., Thiele, C., Jahn, R., 2001. SNAREs are concentrated in cholesterol-dependent clusters that define docking and fusion sites for exocytosis. EMBO J. 20, 2202-2213.

Lewis, M.J., Rayner, J.C., Pelham, H.R.B., 1997. A novel SNARE complex implicated in vesicle fusion with the endoplasmic reticulum. EMBO J. 16, 3017-3024.

Liou, W., Geuze, H.J., Slot, J.W., 1996. Improving structural integrity of cryosections for immunogold labeling. Histochem. Cell Biol. 106, 41-58.

Liu, Y.T., Flanagan, J.J., Barlowe, C., 2004. Sec22p export from the endoplasmic reticulum is independent of SNARE pairing. J. Biol. Chem. 279, 27225-27232.

Majoul, I., Straub, M., Hell, S.W., Duden, R., Söling, H.D., 2001. KDEL-cargo regulates interactions between proteins involved in COPI vesicle traffic: measurements in living cells using FRET. Dev. Cell 1, 139-153.

Malsam, J., Satoh, A., Pelletier, L., Warren, G., 2005. Golgin tethers define subpopulations of COPI vesicles. Science 307 , 1095-1098.

Mann, H.B., Whitney, D.R., 1947. On a test of whether one of two random variables is stochastically larger than the other. Ann. Math. Stat. 18, 50-60.

Marra, P., Maffucci, T., Daniele, T., Di Tullio, G., Ikehara, Y., Chan, E.K.L., Luini, A., Beznoussenko, G., Mironov, A., De Matteis, M.A., 2001. The GM130 and GRASP65 Golgi proteins cycle through and define a subdomain of the intermediate compartment. Nat. Cell Biol. 3, 1101-1113.

McNew, J.A., Parlati, F., Fukuda, R., Johnston, R.J., Paz, K., Paumet, F., Sollner, T.H., Rothman, J.E., 2000. Compartmental specificity of cellular membrane fusion encoded in SNARE proteins. Nature 407, 153-159.

Miller, E., Antonny, B., Hamamoto, S., Schekman, R., 2002. Cargo selection into COPII vesicles is driven by the Sec $24 p$ subunit. EMBO J. 21, 6105-6113.

Mossessova, E., Bickford, L.C., Goldberg, J., 2003. SNARE selectivity of the COPII coat. Cell 114, 483-495.

Nagai, T., Ibata, K., Park, E.S., Kubota, M., Mikoshiba, K., Miyawaki, A., 2002. A variant of yellow fluorescent protein with fast and efficient maturation for cell-biological applications. Nat. Biotechnol. 20, 87-90.

Nakajima, K., Hirose, H., Taniguchi, M., Kurashina, H., Arasaki, K., Nagahama, M., Tani, K., Yamamoto, A., Tagaya, M., 2004. Involvement of BNIP1 in apoptosis and endoplasmic reticulum membrane fusion. EMBO J. 23, 3216-3226.

Newman, A.P., Shim, J., Ferro-Novick, S., 1990. BET1, BOS1, and SEC22 are members of a group of interacting yeast genes required for transport from the endoplasmic reticulum to the Golgi complex. Mol. Cell. Biol. 10, 3405-3414.

Okumura, A.J., Hatsuzawa, K., Tamura, T., Nagaya, H., Saeki, K., Okumura, F., Nagao, K., Nishikawa, M., Yoshimura, A., Wada, I., 2006. Involvement of a novel Q-SNARE, D12, in quality control of the endomembrane system. J. Biol. Chem. 281, 4495-4506.

Orci, L., Stamnes, M., Ravazzola, M., Amherdt, M., Perrelet, A., Sollner, T.H., Rothman, J.E., 1997. Bidirectional transport by distinct populations of COPI-coated vesicles. Cell 90, 335-349.

Rein, U., Andag, U., Duden, R., Schmitt, H.D., Spang, A., 2002. ARF-GAP-mediated interaction between the ERGolgi v-SNAREs and the COPI coat. J. Cell Biol. 157, 395-404.

Sieber, J.J., Willig, K.I., Kutzner, C., Gerding-Reimers, C., Harke, B., Donnert, G., Rammner, B., Eggeling, C., Hell, S.W., Grubmuller, H., Lang, T., 2007. Anatomy and dynamics of a supramolecular membrane protein cluster. Science 317, 1072-1076.

Sohn, K., Orci, L., Ravazzola, M., Amherdt, M., Bremser, M., Lottspeich, F., Fiedler, K., Helms, J.B., Wieland, F.T., 1996. A major transmembrane protein of Golgi-derived COPI-coated vesicles involved in coatomer binding. J. Cell Biol. 135, 1239-1248.

Sollner, T., Bennett, M.K., Whiteheart, S.W., Scheller, R.H., Rothman, J.E., 1993a. A protein assembly-disassembly pathway in vitro that may correspond to sequential steps of synaptic vesicle docking, activation, and fusion. Cell 75, 409-418.

Sollner, T., Whiteheart, S.W., Brunner, M., ErdjumentBromage, H., Geromanos, S., Tempst, P., Rothman, J.E., 1993b. SNAP receptors implicated in vesicle targeting and fusion. Nature 362, 318-324.

Springer, S., Schekman, R., 1998. Nucleation of COPII vesicular coat complex by endoplasmic reticulum to Golgi vesicle SNAREs. Science 281, 698-700.

Sutton, R.B., Fasshauer, D., Jahn, R., Brunger, A.T., 1998. Crystal structure of a SNARE complex involved in synaptic exocytosis at $2.4 \AA$ resolution. Nature 395, 347-353.

Tokuyasu, K., 1973. A technique for ultracryotomy of cell suspensions and tissues. J. Cell Biol. 57, 551-565.

Ungermann, C., Langosch, D., 2005. Functions of SNAREs in intracellular membrane fusion and lipid bilayer mixing. J. Cell Sci. 118, 3819-3828.

Volpicelli-Daley, L.A., Li, Y., Zhang, C.J., Kahn, R.A., 2005. Isoform-selective effects of the depletion of ADP-ribosylation factors 1-5 on membrane traffic. Mol. Biol. Cell 16, 4495-4508.

Weimbs, T., Low, S.H., Chapin, S.J., Mostov, K.E., Bucher, P., Hofmann, K., 1997. A conserved domain is present in different families of vesicular fusion proteins: a new superfamily. Proc. Natl. Acad. Sci. USA 94, 3046-3051.

Wenzel, D., Schauermann, G., von Lupke, A., Hinz, G., 2005. The cargo in vacuolar storage protein transport vesicles is stratified. Traffic 6, 45-55.

Wessel, D., Flügge, U.I., 1984. A method for the quantitative recovery of proteins in dilute solutions in the presence of detergents and lipids. Anal. Biochem. 138, 141-143. 
Xia, Z., Liu, Y., 2001. Reliable and global measurement of fluorescence resonance energy transfer using fluorescence microscopes. Biophys. J. 81, 2395-2402.

Xu, D.L., Joglekar, A.P., Williams, A.L., Hay, J.C., 2000. Subunit structure of a mammalian ER/Golgi SNARE complex. J. Biol. Chem. 275, 39631-39639.

Yasuda, M., Chinnadurai, G., 2000. Functional identification of the apoptosis effector $\mathrm{BH} 3$ domain in cellular protein BNIP1. Oncogene 19, 2363-2367.

Zhang, T., Wong, S.H., Tang, B.L., Xu, Y., Peter, F., Subramaniam, V.N., Hong, W., 1997. The mammalian protein (rbet1) homologous to yeast Betlp is primarily associated with the pre-Golgi intermediate compartment and is involved in vesicular transport from the endoplasmic reticulum to the Golgi apparatus. J. Cell Biol. 139, 1157-1168.

Zhang, T., Wong, S.H., Tang, B.L., Xu, Y., Hong, W.J., 1999. Morphological and functional association of Sec22b/ERS-24 with the pre-Golgi intermediate compartment. Mol. Biol. Cell 10, 435-453.

Zimmermann, T., Rietdorf, J., Girod, A., Georget, V., Pepperkok, R., 2002. Spectral imaging and linear unmixing enables improved FRET efficiency with a novel GFP2-YFP FRET pair. FEBS Lett. 531, 245-249. 